\title{
A mathematical model of HIV infection: The role of $\mathrm{CD8}^{+}$ lymphocytes
}

\author{
Tomáš Hraba \\ Institute of Molecular Genetics \\ Academy of Sciences of the Czech Republic CZ-16637 Prague, Czech \\ Republic. Tel: 4223312282. Fax: 42224310955. \\ e-mail: hraba@img.cas.cz \\ Jaroslav Doležal \\ Institute of Information Theory and Automation \\ Academy of Sciences of the Czech Republic, CZ-18208 Prague, Czech \\ Republic. Tel: 42266052062. Fax: 422824755. \\ e-mail: dolezal@utia.cas.cz
}

\begin{abstract}
The previously developed mathematical model of $\mathrm{CD} 4^{+}$lymphocyte dynamics in HIV infection simulated the respective lymphocyte dynamics well, however simulation of $\mathrm{CD}^{+}$ lymphocyte values was not satisfactory. Modifications of the model were attempted and satisfactory results were obtained, if the influx of immature $\mathrm{CD} 4^{+}$and $\mathrm{CD} 8^{+}$lymphocytes was assumed to be constrained by HIV infection.
\end{abstract}

\section{Keywords}

Mathematical model, HIV infection, $\mathrm{CD} 4^{+}$lymphocyte depletion, $\mathrm{CD} 8^{+}$lymphocyte dynamics

\section{INTRODUCTION}

Our mathematical model of CD4 $4^{+}$lymphocyte dynamics in HIV infection (Hraba et al., 1990) assumes a helper T cell-dependent immune reaction limiting HIV proliferation. It simulates successfully the dynamics of $\mathrm{CD}^{+}$lymphocyte depletion not only qualitatively, but fits the observed data quite well (Doležal and Hraba, 1991).

As $\mathrm{CD}^{+}$lymphocyte numbers are a good prognostic indicator of HIV infection, the model is suitable for simulations of different therapeutic treatments. We have used the mathematical model for simulating the effects of zinovudine therapy on this infection (Hraba and Doležal, 1994), immunotherapy (Doležal and Hraba, 1994a) and of anti-CD8 
antibody administration (Hraba and Doležal, 1995a). The latest treatment was suggested (Adleman and Wofsy, 1993), because it seemed highly probable that a homeostatic mechanism regulating $\mathrm{T}$ cell numbers did not discriminate between the loss of $\mathrm{CD} 4^{+}$or $\mathrm{CD} 8^{+}$ lymphocytes. It was therefore assumed that activation of this mechanism by CD4 $4^{+}$lymphocyte depletion might be inhibited in HIV-infected individuals by increased numbers of $\mathrm{CD} 8^{+}$cells. In consequence, it was suggested to lower the $\mathrm{CD} 8^{+}$cell numbers by administering anti-CD8 antibodies, and in this way, to increase CD4 ${ }^{+}$lymphocyte level by a stronger influx of new $\mathrm{T}$ cells. The model could simulate the effect of anti-CD8 antibody administration, because it incorporated such homeostatic mechanism regulating $\mathrm{T}$ cell level (Hraba et al., 1990).

Simulations carried out with the originally described model showed that CD4 ${ }^{+}$lymphocyte depletion was paralleled by $\mathrm{CD}^{+}$cell number increase (Hraba et al., 1990). Detailed studies were carried out to compare simulated CD4 ${ }^{+}$lymphocyte dynamics with clinical data (Doležal and Hraba, 1991). At that time no analogous attempts were made as far as $\mathrm{CD} 8^{+}$lymphocytes were concerned. Quantitative comparisons of simulated and observed $\mathrm{CD} 8^{+}$lymphocyte dynamics are carried out in this paper.

\section{MODEL}

The model considers immature and mature $\mathrm{CD} 4^{+}(\bar{P}$ and $P$ cells $)$ and $\mathrm{CD} 8^{+}$lymphocytes ( $\bar{R}$ and $R$ cells). As normal values of $R$ cells equal approximately $2 / 3$ of those of $P$ cells, it is assumed that also normal $\bar{R}$ values correspond in a similar way to $2 / 3$ of $\bar{P}$ cells. The sizes of these cell compartments at time $t$ are described by Eqs (1)-(4). The amount of HIV products at time $t$ is given by Eq. (5). Finally, Eq. (6) gives the number of cytotoxic $\mathrm{T}$ cells specific for HIV ( $C$ cells) at time $t$. In the model used in this paper, and in the preceding ones simulating therapeutic interventions in HIV infection, these cells both limit proliferation of HIV, as indicated in Eq. (5), and effect destruction of $\mathrm{CD}^{+}$cells presenting HIV products according to Eqs (1)-(2).

$$
\begin{aligned}
& \frac{d \bar{P}(t)}{d t}=\frac{I_{P}+f\left[\left(P_{0}-P(t)\right)+\left(R_{0}-R(t)\right)\right]}{d(t)}-\bar{\tau}_{P} \bar{P}(t)-\bar{c}_{P} a(t) C(t) \bar{P}(t), \\
& \bar{P}(0)=\bar{P}_{0}
\end{aligned}
$$


where the influx constraining function was

$d(t)= \begin{cases}1 & \text { if } \ln \frac{a(t)}{a_{0}}<L \\ h \ln \frac{a(t)}{a_{0}} & \text { if } \ln \frac{a(t)}{a_{0}} \geq L\end{cases}$

Here $I_{P}$ is the influx of $\bar{P}$ cells, i.e. the rate (all rates are in days ${ }^{-1}$ ) of differentiation of $\bar{P}$ cells from stem cells, $\bar{\tau}_{P}$ is the rate of maturation of $\bar{P}$ cells into $P$ cells, and $\tau_{P}$ is the rate of natural death of $P$ cells; the quantities $\tilde{\tau}_{R}$ and $\tau_{R}$ are defined in a fully analogical way. Further, $f$ is the amplifying coefficient of the linear feedback effect of $P$ and/or $R$ cell decrease on the influx of $\bar{P}$ and $\vec{R}$ cells at time $t$.

The quantity $\bar{c}_{P} a(t) C(t)$ is the rate of elimination of $\bar{P}$ cells due to the amount of HIV products $a(t)$ and the number of cytotoxic T cells $C(t)$ at time $t$. Analogously, $c_{P} a(t) C(t)$ is the rate of elimination of $P$ cells. The value $a_{0}$ is the function of the infectious dose of HIV, $\theta$ characterizes the growth rate of HIV, and $\gamma$ is the rate of inactivation of HIV products mediated by cytotoxic $C$ cells. The maturation of these cells from their precursors is assumed to be dependent on the encounter with HIV products and the effect of HIV specific helper T cells. $I_{C}$ is the influx of $C$ cell precursors, $\varepsilon$ their maturation rate, $\alpha$ the proliferation rate of $C$ cells under the antigenic stimulation by HIV products and helper $\mathrm{T}$ cell influence, and $\tau_{C}$ their natural death rate. Helper $\mathrm{T}$ cell effect on maturation and proliferation of $C$ cells is expressed by the ratio $P(t) / P_{0}$; the coefficient $\nu$ is introduced to characterize the intensity of this helper effect. The value $h$ characterizes HIV-constraining intensity on the $\bar{P}$ and $\bar{R}$ cell influx. Value $L$ defines the level, where such constraining (limiting) effect of $d(t)$ starts.

If not otherwise stated, the model parameters in simulation runs were selected as follows: $\bar{\tau}_{P}=0.2, \tau_{P}=0.01, \vec{\tau}_{R}=0.2, \tau_{R}=0.01, \tau_{C}=0.01, I_{P}=1.0, I_{C}=0.2, \vec{P}_{0}=5.0, P_{0}=$ $100.0, \bar{R}_{0}=3.33, R_{0}=66.7, C_{0}=0.0, a_{0}=0.0005, f=0.01, \alpha=0.3, \varepsilon=0.144, \gamma=$ $0.7, \theta=0.02, \nu=2.0$. Only mature $\mathrm{CD} 4^{+}$lymphocytes were assumed to be susceptible to HIV products, i.e. $\bar{c}_{P}=0.0, c_{P}=20.0$. As a rule, parameter $\varepsilon$ was used for final adjustment of the respective simulation run.

In the subsequent figures numbers of mature CD4 ${ }^{+}$lymphocytes $(P$ cells - solid line) and CD $8^{+}$lymphocytes ( $R$ cells - dashed line) are given as percentage of $P_{0}$. The value of $R_{0}$ is then 66.67 percent. Further, mean observed T cell values in HIV-infected individuals (Lang et al., 1989; Doležal and Hraba, 1991) are depicted: solid circles - CD $4^{+}$ lymphocytes, solid squares - CD8 ${ }^{+}$lymphocytes.

\section{RESULTS}

Clinical data used for quantitative comparisons of simulated and observed CD8 ${ }^{+}$lymphocyte dynamics are those of Lang et al. (1989) which were used for analogous studies with CD4 ${ }^{+}$cells (Doležal and Hraba, 1991). In the above setting such configuration of the model is achieved having $L$ large enough to imply $d(t)=1$ in the model equations (1) and (3), i.e. no influx constraining effect was present.

Simulations of CD8 ${ }^{+}$lymphocyte dynamics using such original model configuration were not satisfactory (Fig. 1). Numbers of these cells increased during the whole course of the infection, when no further increase in these cells was observed clinically, actually, 
it rather seemed that $\mathrm{CD} 8^{+}$cell counts in the patients started to decline in later stages of the infection. No realistic parameter variation was able to change this character of the simulated curve, and it became evident that the model had to be modified to be able to achieve agreement between simulated and observed data. The attempt to improve simulation of $\mathrm{CD} 4^{+}$lymphocyte dynamics by assuming that immature $\mathrm{CD} 8^{+}$lymphocytes were susceptible to HIV products in a similar way as the $\mathrm{CD}^{+}$cells was not successful (Hraba and Doležal, 1995b).

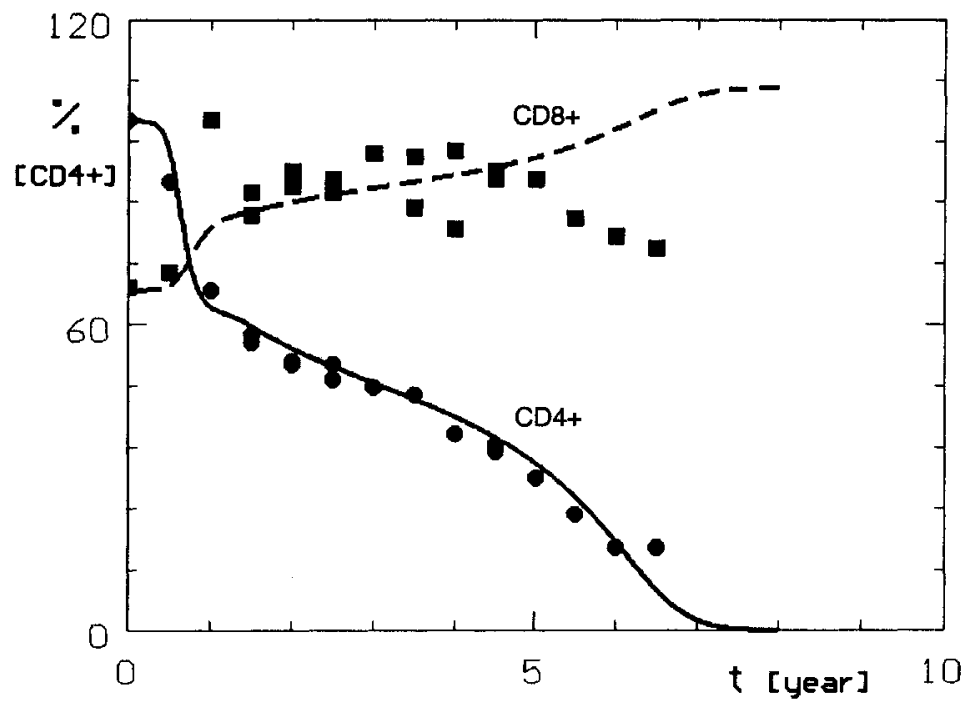

Figure 1 Mature $\mathrm{CD} 4^{+}$and $\mathrm{CD} 8^{+}$lymphocyte dynamics using the original version of the model

We therefore tried to achieve a satisfactory simulation by assuming that HIV infection affects common precursors of $\mathrm{CD} 4^{+}$and $\mathrm{CD} 8^{+}$cells. This situation could be obtained by decreasing the influx of immature $\bar{P}$ and $\bar{R}$ cells due to HIV infection. Simulation results giving best agreement with clinical data were obtained, when the influx was constrained by the logarithm value of HIV product concentration as also given in the definition of $d(t)$ function in Eq. (7). Roughly speaking, such construction allows to control both populations of $T$ cells in intermediate and final phases of the infection.

In Fig. 2, it can be seen that satisfactory agreement between simulated and observed data was obtained under these assumptions with the following changed/additional parameter values: $\alpha=0.7, \varepsilon=0.535, \gamma=0.3, \nu=1.6, h=0.3, L=2.5$. The final abrupt drop of simulated CD8 ${ }^{+}$lymphocyte numbers is only an extrapolation, occurring when patients are assumed to be dying, or are even dead, and the respective virus concentraions reach seemingly biologically not feasible values. 


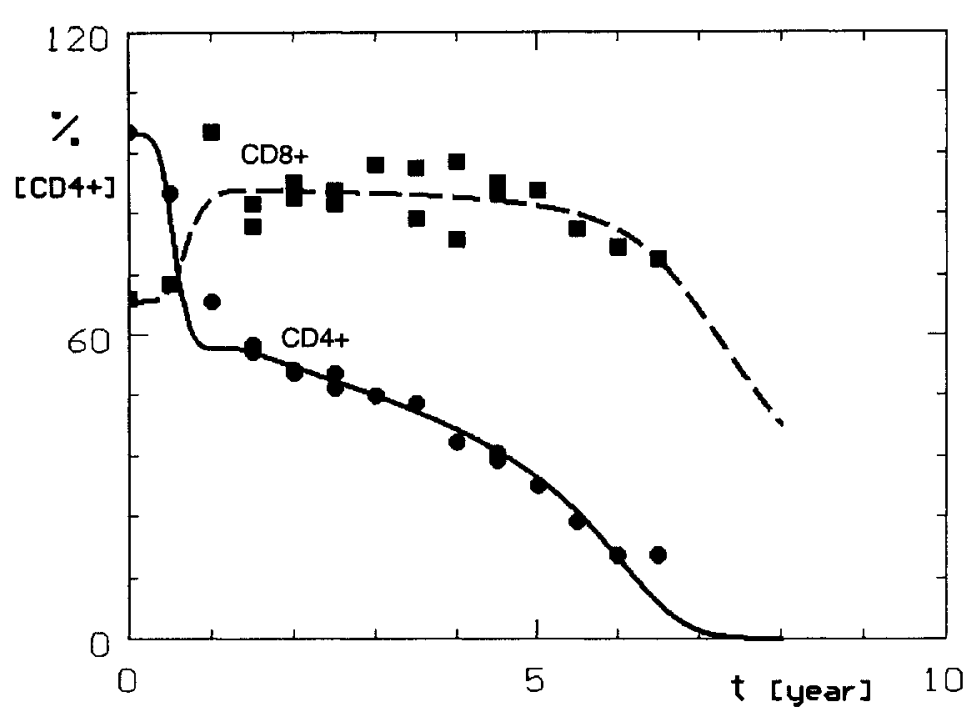

Figure 2 Mature $\mathrm{CD} 4^{+}$and $\mathrm{CD} 8^{+}$lymphocyte dynamics simulated by model modification with HIV-constrained $\mathrm{T}$ cell influx

\section{CONCLUSIONS}

The major advantage of our model is that it is able to simulate reasonably well $\mathrm{CD} 4^{+}$ lymphocyte dynamics in all stages of infection more appropriately than some alternative, more sophisticated models of HIV infection (Nowak et al., 1991; Perelson et al., 1993). The modified version simulates also $\mathrm{CD} 8^{+}$lymphocyte dynamics satisfactorily. The model is rather versatile and easily adaptable to different assumptions. When the model was formulated originally, it was assumed that the cytopathogenic effect of HIV was not responsible for the $\mathrm{CD}^{+}$lymphocyte depletion. It was postulated that HIV products induced this depletion by some other mechanism either directly, e.g. by apoptosis, or indirectly, e.g. by inducing an (auto)immune reaction.

The model version used in this, and most preceding papers, assumes that the specific immune response induced by HIV not only limits HIV proliferation but is also instrumental in $\mathrm{CD}^{+}$lymphocyte elimination in a similar way as it was suggested by Zinkernagel et al. (1994) and recent findings (Ho et al., 1995; Wei et al., 1995). We preferred this version to that in which HIV products were assumed to induce CD4 ${ }^{+}$cell elimination directly, because it gave a better fit to observed CD4 ${ }^{+}$lymphocyte values. However, the difference was not substantial, and in absence of observation data permitting us to determine proper parameter values, we sticked to this arrangement just for its convenience. However, it is possible to adjust the model to any other assumption concerning pathogenic mechanisms, including even direct cytopathogenic effect of HIV on $\mathrm{CD} 4^{+}$lymphocytes. 


\section{REFERENCES}

Adleman, L.M. and Wofsy, D. (1993) T-cell homeostasis: implications in HIV infection. J. AIDS, 6, 144-52.

Doležal, J. and Hraba, T. (1991) Model-based analysis of CD4 ${ }^{+}$lymphocyte dynamics in HIV infected individuals. II. Evaluation of the model based on clinical observations. Immunobiology, 182, 178-87.

Doležal, J. and Hraba, T. (1994) Mathematical modelling of immunotherapy in HIV infection. Folia Biol. (Praha), 40, 193-9.

Ho, D.D., Neumann, A.U., Perelson, A.S., Chen, W., Leonard, J.M. and Markowitz, M (1995) Rapid turnover of plasma virions and CD4 lymphocytes in HIV-1 infection. Nature, 373, 123-6.

Hraba, T. and Doležal, J. (1994) Mathematical modelling of chemotherapy in HIV infection. Folia Biol. (Praha), 40, 103-11.

Hraba, T. and Doležal, J. (1995a) Mathematical modelling of HIV infection therapy. Int. J. Immunopharmacol. In press.

Hraba, T. and Doležal, J. (1995b) Mathematical modelling of CD8 ${ }^{+}$lymphocyte dynamics in HIV infection. Folia Biol. (Praha). In press.

Hraba, T., Doležal, J. and Čelikovský, S. (1990) Model-based analysis of CD4+ lymphocyte dynamics in HIV infected individuals. Immunobiology, 181, 108-18.

Lang, W., Perkins, H., Anderson, R.E., Royce, R., Jewell, N. and Winkelstein, W. (1989) Patterns of $\mathrm{T}$ lymphocyte changes with human immunodeficiency virus infection: from seroconversion to the development of AIDS. J. AIDS, 2, 63-9.

Nowak, M.A., Anderson, R.M., McLean, A.R., Wolfs, T.F.W., Goudsmit, J. and May, R.M. (1991) Antigenic diversity thresholds and the development of AIDS. Science, 254, 963-9.

Perelson, A.S., Kirschner, D.E. and de Boer, R. (1993) Dynamics of HIV infection of CD4 ${ }^{+}$ T cells. Math. Biosci. 114, 81-125.

Wei, X., Ghosh, S.K., Taylor, M.E., Johnson, V.A., Emini, E.A., Deutsch, P., Lifson, J.D., Bonhoeffer, S., Nowak, M.A., Hahn, B.A., Saag, M.S. and Shaw, G.M (1995) Viral dynamics in human immunodeficiency virus type 1 infection. Nature, 373, 117-22.

Zinkernagel, R.M. and Hengartner, H. (1994) T-cell-mediated immunopathology versus direct cytolysis by virus: implications for HIV and AIDS. Immunol. Today, 15, 262-8. 